\title{
SOME RESULTS AND PROBLEMS ON ADVECTION-DIFFUSION EQUATIONS
}

\author{
P. Braz e Silva \\ L. Schütz \\ P. R. Zingano*
}

Dedicated to Professor J. V. Gonçalves on the occasion of his $60^{\text {th }}$ birthday

\begin{abstract}
We describe a Moser-type iteration procedure to derive decay estimates for solutions $u(\cdot, t)$ of very general advection-diffusion equations in $n$-dimensional space, assuming $u(\cdot, 0)=u_{0} \in L^{p}\left(\mathbb{R}^{n}\right), 1 \leq p<\infty$. A number of related results are also discussed.
\end{abstract}

\section{Introduction}

In this work, we consider solutions $u(\cdot, t)$ of the scalar advection-diffusion equation

$$
u_{t}+\operatorname{div}(\boldsymbol{b}(\boldsymbol{x}, t, u) u)=\operatorname{div}(A(\boldsymbol{x}, t, u) \nabla u), \quad \boldsymbol{x} \in \mathbb{R}^{n}, t>0,
$$

with general Cauchy data

$$
u(\cdot, 0)=u_{0} \in L^{p}\left(\mathbb{R}^{n}\right)
$$

for some $1 \leq p<\infty$, where $\boldsymbol{b}$ (the advection velocity field) and $A$ (the viscosity tensor) are given $C^{1}$ functions. (Throughout the text, boldface letters are used for vector quantities, and div denotes the divergence with respect to the space variable $\boldsymbol{x}$.) Condition (1.2) is meant in $L^{p}$, i.e., $\left\|u(\cdot, t) \rightarrow u_{0}\right\|_{L^{p}} \rightarrow 0$ as $t \rightarrow 0$. It is assumed that $A$ satisfies the positive definiteness condition

$$
\langle A(\boldsymbol{x}, t, \mathrm{v}) \boldsymbol{\xi}, \boldsymbol{\xi}\rangle \geq \mu(t)|\boldsymbol{\xi}|^{2}, \quad \forall \boldsymbol{\xi} \in \mathbb{R}^{n},
$$

Mathematics Subject Classification. 35B40 (primary); 35B45, 35K15 (secondary).

Key words and phrases. a priori estimates, decay rates, diffusion waves, heterogeneous media, non-isotropic media, Moser iteration, Nash's inequality, $L^{p}$ behavior.

${ }^{*}$ Work partly supported by CNPq, Brazil 
for all $\boldsymbol{x}, t, \mathrm{v}$ and some positive $\mu \in C^{0}([0, \infty[)$ verifying

$$
\lim _{t \rightarrow \infty} t \mu(t)=\infty
$$

Moreover, $\boldsymbol{b}=\left(b_{1}, \ldots, b_{n}\right)$ must verify

$$
\sum_{j=1}^{n} \frac{\partial b_{j}}{\partial x_{j}}(\boldsymbol{x}, t, \mathrm{v}) \geq 0, \quad \forall \boldsymbol{x} \in \mathbb{R}^{n}, t>0, \mathrm{v} \in \mathbb{R},
$$

or, alternatively, the Carlen-Loss condition [5]

$$
\int_{\mathbb{R}^{n}}|\Phi(\boldsymbol{x})|^{q} \operatorname{div} \boldsymbol{b}(\boldsymbol{x}, t, \Phi(\boldsymbol{x})) d \boldsymbol{x} \geq 0, \quad \forall t>0,
$$

for all $\Phi \in C_{0}^{\infty}\left(\mathbb{R}^{n}\right)$ and $q \geq p$. It is also assumed that $\boldsymbol{b}(\boldsymbol{x}, t, \mathrm{v}), A(\boldsymbol{x}, t, \mathrm{v})$ are bounded for $\boldsymbol{x} \in \mathbb{R}^{n}$ and bounded $t, \mathrm{v} \in \mathbb{R}$, with $\boldsymbol{b}, A$ locally Lipschitz continuous with respect to $\mathrm{v}$. It then follows from classical existence theory and Theorem 2.3 below that there exists a unique solution $u(\cdot, t) \in C^{0}\left(\left[0, \infty\left[, L^{p}\left(\mathbb{R}^{n}\right)\right) \cap\right.\right.$ $L_{1 \mathrm{oc}}^{\infty}(] 0, \infty\left[, L^{\infty}\left(\mathbb{R}^{n}\right)\right)$ to problem (1.1), (1.2), which is the subject of this paper.

An important example is given by the equation

$$
u_{t}+\operatorname{div}(\boldsymbol{b}(u) u)=\Delta u, \quad \boldsymbol{x} \in \mathbb{R}^{n}, t>0
$$

which has been studied by several authors (see e.g. [5, 7, 8, 16, 17] and references therein), under further assumptions on the advection term or the initial data, using logarithmic Sobolev inequalities, Fourier splitting, or other techniques. Our approach is more elementary and is based on the fundamental fact that $\|u(\cdot, t)\|_{L^{2 q}\left(\mathbb{R}^{n}\right)}$ can be bounded in terms of $\|u(\cdot, t)\|_{L^{q}\left(\mathbb{R}^{n}\right)}$, which suggests a simple iterative procedure to estimate the supnorm $\|u(\cdot, t)\|_{L^{\infty}\left(\mathbb{R}^{n}\right)}$ analogous to the familiar Moser iteration for elliptic equations [12, 13]. This method is easy to extend to more general equations such as (1.1), and can be adapted (with corresponding change in results) to other problems as well, including equations with bounded velocities $\boldsymbol{b}$ not verifying (1.5), equations with source terms, degenerate problems with $\mu(t)$ vanishing at isolated zeros, and 
other examples $[3,18]$.

In particular, it follows from Theorem 2.3 below that one gets

$$
\|u(\cdot, t)\|_{L^{q}\left(\mathbb{R}^{n}\right)} \leq K(n, p, q)\left\|u_{0}\right\|_{L^{p}\left(\mathbb{R}^{n}\right)}\left(\mu_{0} t\right)^{-\frac{n}{2}\left(\frac{1}{p}-\frac{1}{q}\right)}, \quad \forall t>0, p \leq q \leq \infty,
$$

when $\mu(t) \geq \mu_{0}>0$, as in equation (1.6), for some constant $K$ depending on $n, p, q$ but not on $\boldsymbol{b}, A, u_{0}$. Similar estimates are obtained for general $\mu(t)$ satisfying (1.4) above, yielding $\|u(\cdot, t)\|_{L^{q}} \rightarrow 0$ as $t \rightarrow \infty$ for all $q>p$, and also

$$
\lim _{t \rightarrow \infty}\|u(\cdot, t)\|_{L^{p}\left(\mathbb{R}^{n}\right)}=0
$$

when $p>1$. On the other hand, if $p=1$, the time asymptotic value of $\|u(\cdot, t)\|_{L^{1}\left(\mathbb{R}^{n}\right)}$ for $t \gg 1$ is not known in general. For special equations such as (1.6), it has been shown that $\|u(\cdot, t)\|_{L^{1}\left(\mathbb{R}^{n}\right)} \rightarrow|m|$ for any solution, where $m$ denotes its (time-invariant) mass, see e.g. [2, 15, 22]. However, this simple result does not hold for arbitrary solutions of (1.1) in general, and characterizing which equations (1.1) do satisfy this property remains open. Some related problems and results are briefly discussed in Section 3.

\section{Some a priori estimates.}

In this section we derive the main estimates, including some of their consequences. To make the argument more transparent, we will assume in addition that $\mu(t)$ is monotonically non-increasing as a function of $t$. (This can always be achieved by redefining $\mu(t)$ for each $t$ as the minimum value up to time $t$; note that (1.4) remains valid if $\mu(t)$ is redefined this way.) The case of general $\mu(t)$ is more technical and will be discussed in a forthcoming paper. Also, it will be convenient to use regularized sign functions $L_{\delta}^{\prime}$ (see e.g. [10], p. 533): picking (any) $S \in C^{1}(\mathbb{R})$ monotonically nondecreasing with $S(0)=0, S(\mathrm{v})=1$ for all $\mathrm{v} \geq 1$ and $S(\mathrm{v})=-1$ for all $\mathrm{v} \leq-1$, we set, for arbitrary $\delta>0$,

$$
L_{\delta}(\mathrm{v}):=\delta \int_{0}^{\mathrm{v} / \delta} S(\mathrm{w}) d \mathrm{w}, \quad \mathrm{v} \in \mathbb{R},
$$


so that $L_{\delta}(\mathrm{v}) \rightarrow|\mathrm{v}|, L_{\delta}^{\prime}(\mathrm{v}) \rightarrow \operatorname{sgn} \mathrm{v}$ as $\delta \rightarrow 0$.

Lemma 2.1. For any $q \geq p,\|u(\cdot, t)\|_{L^{q}\left(\mathbb{R}^{n}\right)} \leq\left\|u\left(\cdot, t_{0}\right)\right\|_{L^{q}\left(\mathbb{R}^{n}\right)} \forall t>t_{0} \geq 0$.

Proof: Let $\Phi_{\delta}(\mathrm{v}):=L_{\delta}(\mathrm{v})^{q}$. Assuming $\left\|u\left(\cdot, t_{0}\right)\right\|_{L^{q}\left(\mathbb{R}^{n}\right)}$ finite, we obtain, multiplying (1.1) by $\Phi_{\delta}^{\prime}(u(\boldsymbol{x}, t))$ and integrating on $\mathbb{R}^{n} \times\left[t_{0}, t\right]$,

$$
\begin{aligned}
& \int_{\mathbb{R}^{n}} L_{\delta}(u(\boldsymbol{x}, t))^{q} d \boldsymbol{x}+\int_{t_{0}}^{t} \int_{\mathbb{R}^{n}} \Phi_{\delta}^{\prime \prime}(u(\boldsymbol{x}, \tau))\langle A(\boldsymbol{x}, \tau, u) \nabla u, \nabla u\rangle d \boldsymbol{x} d \tau= \\
= & \int_{\mathbb{R}^{n}} L_{\delta}\left(u\left(\boldsymbol{x}, t_{0}\right)\right)^{q} d \boldsymbol{x}+\int_{t_{0}}^{t} \int_{\mathbb{R}^{n}} \Phi_{\delta}^{\prime \prime}(u(\boldsymbol{x}, \tau)) u(\boldsymbol{x}, \tau)\langle\boldsymbol{b}(\boldsymbol{x}, \tau, u), \nabla u\rangle d \boldsymbol{x} d \tau .
\end{aligned}
$$

By (1.3), we then have

$$
\begin{aligned}
& \int_{\mathbb{R}^{n}} L_{\delta}(u(\boldsymbol{x}, t))^{q} d \boldsymbol{x} \leq \int_{\mathbb{R}^{r}} L_{\delta}\left(u\left(\boldsymbol{x}, t_{0}\right)\right)^{q} d \boldsymbol{x} \\
& \quad+\int_{t_{0}}^{t} \int_{\mathbb{R}^{n}} \Phi_{\delta}^{\prime \prime}(u(\boldsymbol{x}, \tau)) u(\boldsymbol{x}, \tau)\langle\boldsymbol{b}(\boldsymbol{x}, \tau, u), \nabla u\rangle d \boldsymbol{x} d \tau,
\end{aligned}
$$

since $\Phi_{\delta}^{\prime \prime}(\mathrm{v})=q(q-1) L_{\delta}(\mathrm{v})^{q-2} L_{\delta}^{\prime}(\mathrm{v})^{2}+q L_{\delta}(\mathrm{v})^{q-1} L_{\delta}^{\prime \prime}(\mathrm{v}) \geq 0$. Letting $\delta \rightarrow$ 0 , we have $\mathrm{v} L_{\delta}^{\prime \prime}(\mathrm{v}) \rightarrow 0, L_{\delta}(\mathrm{v})^{q-2} L_{\delta}^{\prime}(\mathrm{v})^{2} \mathrm{v} \rightarrow|\mathrm{v}|^{q-1} \operatorname{sgn}(\mathrm{v}), L_{\delta}(\mathrm{v})^{q} \rightarrow|\mathrm{v}|^{q}$ for each $v \in \mathbb{R}$, so that, by Lebesgue's dominated convergence theorem, we get from the estimate above, as $\delta \rightarrow 0$,

$$
\|u(\cdot, t)\|_{L^{q}\left(\mathbb{R}^{n}\right)}^{q} \leq\left\|u\left(\cdot, t_{0}\right)\right\|_{L^{q}\left(\mathbb{R}^{n}\right)}^{q}+E_{q}(t)
$$

where

$$
E_{q}(t)=q(q-1) \int_{t_{0}}^{t} \int_{\mathbb{R}^{n}}|u(\boldsymbol{x}, \tau)|^{q-1} \operatorname{sgn}(u(\boldsymbol{x}, \tau))\langle\boldsymbol{b}(\boldsymbol{x}, \tau, u), \nabla u\rangle d \boldsymbol{x} d \tau .
$$

The result now follows, since $E_{q}(t)=0$ for $q=1$ and, for $q>1$,

$$
\begin{aligned}
& \frac{E_{q}(t)}{q-1}=-\int_{t_{0}}^{t} \int_{\mathbb{R}^{n}}|u(\boldsymbol{x}, \tau)|^{q} \operatorname{div} \boldsymbol{b}(\boldsymbol{x}, \tau, u) d \boldsymbol{x} d \tau \\
& =-q \int_{t_{0}}^{t} \int_{\mathbb{R}^{n}} \int_{0}^{u(\boldsymbol{x}, \tau)} \operatorname{sgn}(\mathrm{v})|\mathrm{v}|^{q-1} \sum_{j=1}^{n} \frac{\partial b_{j}}{\partial x_{j}}(\boldsymbol{x}, \tau, \mathrm{v}) d \mathrm{v} d \boldsymbol{x} d \tau,
\end{aligned}
$$


so that $E_{q}(t) \leq 0$ in view of $(1.5 \mathrm{a})$ or $(1.5 \mathrm{~b})$.

In particular, the maximum principle $\|u(\cdot, t)\|_{L^{\infty}\left(\mathbb{R}^{n}\right)} \leq\left\|u_{0}\right\|_{L^{\infty}\left(\mathbb{R}^{n}\right)}$ is valid for all $t>0$.

Theorem 2.2. For any $q \geq p$,

$$
\|u(\cdot, t)\|_{L^{2 q}\left(\mathbb{R}^{n}\right)} \leq K_{1}(q, n)\left\|u\left(\cdot, t_{0}\right)\right\|_{L^{q}\left(\mathbb{R}^{n}\right)}\left(\left(t-t_{0}\right) \mu(t)\right)^{-\frac{n}{4 q}}
$$

for all $t>t_{0} \geq 0$, where

$$
K_{1}(q, n)=\left\{2^{-n} C_{n}^{n+2}\left(1+\frac{n}{2}\right)^{1+\frac{n}{2}}\left(1-\frac{1}{2 q}\right)^{-\frac{n}{2}}\right\}^{\frac{1}{2 q}} .
$$

In (2.2), $C_{n}$ denotes the constant in the Nirenberg-Gagliardo inequality (see e.g. [9], p. 24)

$$
\|\mathrm{w}\|_{L^{2}\left(\mathbb{R}^{n}\right)} \leq C_{n}\|\mathrm{w}\|_{L^{1}\left(\mathbb{R}^{n}\right)}^{\frac{2}{n+2}}\|\nabla \mathrm{w}\|_{L^{2}\left(\mathbb{R}^{n}\right)}^{\frac{n}{n+2}}, \quad \mathrm{w} \in H^{1}\left(\mathbb{R}^{n}\right) \cap L^{1}\left(\mathbb{R}^{n}\right) .
$$

This particular inequality was originally obtained by Nash [14], with optimal values for $C_{n}$ determined later in [4]. The following proof of Theorem 2.2 extends an argument introduced in [20] and further developed in [19, 21, 2].

Proof: Multiplying (1.1) by $\left(t-t_{0}\right)^{\gamma} \Phi_{\delta}^{\prime}(u(\boldsymbol{x}, t)), \gamma=1+\frac{n}{2}, \Phi_{\delta}(\mathrm{v}):=L_{\delta}(\mathrm{v})^{2 q}$, with $L_{\delta}(\cdot)$ given in (2.1), and integrating on $\mathbb{R}^{n} \times\left[t_{0}, t\right]$, we obtain, integrating by parts,

$$
\begin{aligned}
& \left(t-t_{0}\right)^{\gamma} \int_{\mathbb{R}^{n}} L_{\delta}(u(\boldsymbol{x}, t))^{2 q} d \boldsymbol{x} \\
& +2 q(2 q-1) \int_{t_{0}}^{t}\left(\tau-t_{0}\right)^{\gamma} \mu(\tau) \int_{\mathbb{R}^{n}} L_{\delta}(u(\boldsymbol{x}, \tau))^{2 q-2} L_{\delta}^{\prime}(u)^{2}|\nabla u|^{2} d \boldsymbol{x} d \tau \\
& \leq \gamma \int_{t_{0}}^{t}\left(\tau-t_{0}\right)^{\gamma-1} \int_{\mathbb{R}^{n}} L_{\delta}(u(\boldsymbol{x}, \tau))^{2 q} d \boldsymbol{x} d \tau \\
& \quad+\int_{t_{0}}^{t}\left(\tau-t_{0}\right)^{\gamma} \int_{\mathbb{R}^{n}} \Phi_{\delta}^{\prime \prime}(u) u(\boldsymbol{x}, \tau)\langle\boldsymbol{b}(\boldsymbol{x}, \tau, u), \nabla u\rangle d \boldsymbol{x} d \tau
\end{aligned}
$$


using (1.3) and the fact that $L_{\delta}^{\prime \prime}(\mathrm{v}) \geq 0$. By (1.5), we then get, as in the previous proof, letting $\delta \rightarrow 0$,

$$
\mathbb{E}_{q}(t) \leq\left(1+\frac{n}{2}\right) \int_{t_{0}}^{t}\left(\tau-t_{0}\right)^{\gamma-1}\|u(\cdot, \tau)\|_{L^{2 q}\left(\mathbb{R}^{n}\right)}^{2 q} d \tau
$$

where

$$
\begin{aligned}
\mathbb{E}_{q}(t):= & \left(t-t_{0}\right)^{\gamma}\|u(\cdot, t)\|_{L^{2 q}\left(\mathbb{R}^{n}\right)}^{2 q} \\
& +\left.2 q(2 q-1) \mu(t) \int_{t_{0}}^{t}\left(\tau-t_{0}\right)^{\gamma} \int_{\mathbb{R}^{n}} u(\boldsymbol{x}, \tau)\right|^{2 q-2}|\nabla u|^{2} d \boldsymbol{x} d \tau .
\end{aligned}
$$

Applying (2.3) to $\mathrm{w}=|u|^{q}$, we get

$$
\|u(\cdot, \tau)\|_{L^{2 q}\left(\mathbb{R}^{n}\right)}^{2 q} \leq q^{\frac{2 n}{n+2}} C_{n}^{2}\|u(\cdot, \tau)\|_{L^{q}\left(\mathbb{R}^{n}\right)}^{\frac{4 q}{n+2}}\left(\int_{\mathbb{R}^{n}}|u(\boldsymbol{x}, \tau)|^{2 q-2}|\nabla u(\boldsymbol{x}, \tau)|^{2} d \boldsymbol{x}\right)^{\frac{n}{n+2}},
$$

so that we obtain, using Hölder's inequality,

$\mathbb{E}_{q}(t) \leq 4^{-\frac{n}{n+2}}\left(1+\frac{n}{2}\right)\left(1-\frac{1}{2 q}\right)^{-\frac{n}{n+2}} C_{n}^{2}\left(\tau-t_{0}\right)^{\frac{2}{n+2}} \mu(t)^{-\frac{n}{n+2}} \| u\left(\cdot, t_{0} \|_{L^{q}\left(\mathbb{R}^{n}\right)}^{\frac{4 q}{n+2}} \mathrm{E}_{q}(t)^{\frac{n}{n+2}}\right.$

that is,

$$
\begin{aligned}
& \left(t-t_{0}\right)^{\frac{n}{2}}\|u(\cdot, t)\|_{L^{2 q}\left(\mathbb{R}^{n}\right)}^{2 q}+ \\
& \quad+2 q(2 q-1) \frac{\mu(t)}{t-t_{0}} \int_{t_{0}}^{t}\left(\tau-t_{0}\right)^{1+\frac{n}{2}} \int_{\mathbb{R}^{n}}|u(\boldsymbol{x}, \tau)|^{2 q-2}|\nabla u|^{2} d \boldsymbol{x} d \tau \leq \\
& \quad \leq 2^{-n}\left(1+\frac{n}{2}\right)^{1+\frac{n}{2}}\left(1-\frac{1}{2 q}\right)^{-\frac{n}{2}} C_{n}^{n+2} \mu(t)^{-\frac{n}{2}}\left\|u\left(\cdot, t_{0}\right)\right\|_{L^{q}\left(\mathbb{R}^{n}\right)}^{2 q} .
\end{aligned}
$$

We are now in good standing to establish our main result, given in Theorem 2.3 below.

Theorem 2.3. $\quad\|u(\cdot, t)\|_{L^{\infty}\left(\mathbb{R}^{n}\right)} \leq K(n, p)\left\|u_{0}\right\|_{L^{p}\left(\mathbb{R}^{n}\right)}(\mu(t) t)^{-\frac{n}{2 p}}$ for all $t>0$, where

$$
K(n, p)=\left(\frac{n}{2}+1\right)^{\frac{1}{p}\left(\frac{n}{2}+1\right)} C_{n}^{\frac{n+2}{p}}\left[\prod_{i=1}^{\infty}\left(1-\frac{1}{2^{i} p}\right)^{\frac{1}{2^{i} p}}\right]^{-\frac{n}{2}}
$$


Remark: In (2.4), $C_{n}$ is the constant given in Nash's inequality (2.3). The value (2.4) is not optimal; minimal values for $K(n, p)$ are not actually known, except in special cases.

Proof: By Moser iteration [12, 13], as follows: for $k \in \mathbb{N}$, we set $t_{0}:=2^{-k} t$, $t_{i}:=t_{i-1}+2^{-i} t, 1 \leq i \leq k$, so that $t_{k}=t$ and, from Theorem 2.2 above,

$$
\begin{aligned}
\left\|u\left(\cdot, t_{i}\right)\right\|_{L^{2^{i} p}\left(\mathbb{R}^{n}\right)} & \leq \\
& \leq \hat{K}(n)^{\frac{1}{2^{i} p}} \Gamma\left(n, 2^{i} p\right)^{\frac{1}{2^{i} p}} \mu\left(t_{i}\right)^{-\frac{n}{2^{i+1} p}}\left(2^{-i} t\right)^{-\frac{n}{2^{i+1} p}}\left\|u\left(\cdot, t_{i-1}\right)\right\|_{L^{2^{i-1} p}\left(\mathbb{R}^{n}\right)}
\end{aligned}
$$

for every $1 \leq i \leq k$, where $\hat{K}(n)=2^{-n}(n / 2+1)^{n / 2+1} C_{n}^{n+2}$ and $\Gamma(n, q)=$ $(1-1 / q)^{-n / 2}$. This gives, for non-increasing $\mu(t)$,

$$
\begin{aligned}
& \|u(\cdot, t)\|_{L^{2^{k_{p}}}\left(\mathbb{R}^{n}\right)} \leq \\
& \quad \leq \hat{K}(n)^{\frac{1}{p} \lambda_{k}}(\mu(t) t)^{-\frac{n}{2 p} \lambda_{k}}\left[\prod_{i=1}^{k}\left(1-\frac{1}{2^{i} p}\right)^{\frac{1}{2^{i} p}} 2^{-\frac{i}{2^{i} p}}\right]^{-\frac{n}{2}}\left\|u\left(\cdot, t_{0}\right)\right\|_{L^{p}\left(\mathbb{R}^{n}\right)},
\end{aligned}
$$

where $\lambda_{k}=1-2^{-k}$. Letting $k \rightarrow \infty$, the result is obtained.

From Lemma 2.1 and Theorem 2.3, we then obtain the following result by interpolation.

Corollary 2.4. For any $q \geq p$,

$$
\|u(\cdot, t)\|_{L^{q}\left(\mathbb{R}^{n}\right)} \leq K(n, p)^{1-\frac{p}{q}}\left\|u_{0}\right\|_{L^{p}\left(\mathbb{R}^{n}\right)}(\mu(t) t)^{-\frac{n}{2}\left(\frac{1}{p}-\frac{1}{q}\right)}
$$

for all $t>0$, where $K(n, p)$ is given in (2.4). 
Using standard estimates for fundamental solutions of linear parabolic problems $[1,11]$, decay estimates for derivatives of $u(\cdot, t)$ can now be obtained. For example, given $t_{0}>0$, one gets, for $p \leq q \leq \infty$,

$\|\nabla u(\cdot, t)\|_{L^{q}\left(\mathbb{R}^{n}\right)} \leq K\left(n, p, q, t_{0}\right)\left\|u_{0}\right\|_{L^{p}\left(\mathbb{R}^{n}\right)}(\mu(t) t)^{-\frac{n}{2}\left(\frac{1}{p}-\frac{1}{q}\right)-\frac{1}{2}}, \quad \forall t>t_{0}$,

where constant $K\left(n, p, q, t_{0}\right)$ depends on $n, p, q, t_{0}$, the magnitude of $\left\|u_{0}\right\|_{L^{p}\left(\mathbb{R}^{n}\right)}$ and the functions $\boldsymbol{b}, A$. We thus see that, assuming $(1.4), u(\cdot, t)$ and its derivatives decay time asymptotically in $L^{q}$ for any $q \geq p$, except (possibly) for the

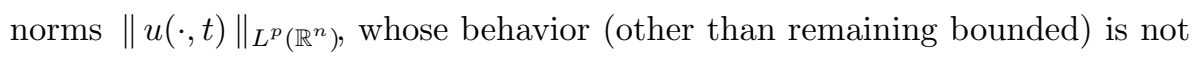
immediately clear from Theorem 2.3. This question will be briefly examined below.

\section{Behavior of $\|u(\cdot, t)\|_{L^{p}\left(\mathbb{R}^{n}\right)}$.}

When $p>1$, the answer turns out to be very simple.

Theorem 3.1. If $p>1$, then $\lim _{t \rightarrow \infty}\|u(\cdot, t)\|_{L^{p}\left(\mathbb{R}^{n}\right)}=0$.

Proof: Given $\varepsilon>0$, let $R \gg 1$ be such that $\left\|v_{0}^{(R)}\right\|_{L^{p}\left(\mathbb{R}^{n}\right)} \leq \varepsilon$, where $v_{0}^{(R)}(\boldsymbol{x}):=u_{0}(\boldsymbol{x})$ if $|\boldsymbol{x}|>R$ and zero otherwise. Setting $v^{(R)}(\cdot, t)$ to be the solution of equation (1.1) with initial data $v^{(R)}(\cdot, 0)=v_{0}^{(R)}$, then

$$
\begin{aligned}
\|u(\cdot, t)\|_{L^{p}\left(\mathbb{R}^{n}\right)} & \leq\left\|u(\cdot, t)-v^{(R)}(\cdot, t)\right\|_{L^{p}\left(\mathbb{R}^{n}\right)}+\varepsilon \\
& \leq\left\|u_{0}-v_{0}^{(R)}\right\|_{L^{1}\left(\mathbb{R}^{n}\right)}^{1 / p}\left\|u(\cdot, t)-v^{(R)}(\cdot, t)\right\|_{L^{\infty}\left(\mathbb{R}^{n}\right)}^{1-1 / p}+\varepsilon,
\end{aligned}
$$

because (1.1) is $L^{1}$-contracting, see e.g. [10]. Therefore,

$$
\|u(\cdot, t)\|_{L^{p}\left(\mathbb{R}^{n}\right)} \leq C(p, n, R)\left\|u_{0}\right\|_{L^{p}\left(\mathbb{R}^{n}\right)}(\mu(t) t)^{-\frac{n}{2 p}\left(1-\frac{1}{p}\right)}+\varepsilon
$$


for some quantity $C(p, n, R)$ independent of $t$, by Theorem 2.3, and so $\|u(\cdot, t)\|_{L^{p}\left(\mathbb{R}^{n}\right)} \leq 2 \varepsilon$ for $t \gg 1$.

Corollary 3.2. If $1<p \leq q \leq \infty$, then we have $\lim _{t \rightarrow \infty}(\mu(t) t)^{\frac{n}{2}\left(\frac{1}{p}-\frac{1}{q}\right)}\|u(\cdot, t)\|_{L^{q}\left(\mathbb{R}^{n}\right)}=0, \quad$ uniformly in $q$.

Thus, if $p>1$, solutions decay actually faster than the rates obtained above. As with the familiar case of heat equation, however, this extra speed depends on particular features of the initial state $u_{0} \in L^{p}\left(\mathbb{R}^{n}\right)$ and can be arbitrarily slow, so that no higher rates are valid in general. In this sense, therefore, our results are optimal.

Proof: Because

$$
\begin{aligned}
& (\mu(t) t)^{\frac{n}{2 p}}\|u(\cdot, t)\|_{L^{\infty}\left(\mathbb{R}^{n}\right)} \leq \\
& \quad \leq C_{n, p}\left((\mu(t) t)^{\frac{n}{2 p}+\frac{1}{2}}\|\nabla u(\cdot, t)\|_{L^{\infty}\left(\mathbb{R}^{n}\right)}\right)^{\frac{n}{n+p}}\left(\|u(\cdot, t)\|_{L^{p}\left(\mathbb{R}^{n}\right)}\right)^{\frac{p}{n+p}}
\end{aligned}
$$

for some appropriate Sobolev constant $C_{n, p}>0$ (see e.g. [9], Theorem 9.3), the result holds for $q=\infty$ by (2.5) and Theorem 3.1 above. Extension to arbitrary $q>p$ then follows directly from Theorem 3.1 and interpolation.

On the other hand, in case $p=1$, the limiting value of $\|u(\cdot, t)\|_{L^{1}\left(\mathbb{R}^{n}\right)}$ as $t \rightarrow \infty$ is not known in general. Important special features in this case include the conservation of solution mass, that is,

$$
m=\int_{\mathbb{R}^{n}} u_{0}(\boldsymbol{x}) d \boldsymbol{x}=\int_{\mathbb{R}^{n}} u(\boldsymbol{x}, t) d \boldsymbol{x}, \quad \forall t>t_{0},
$$

and the monotonicity property that $u(\cdot, t) \geq \tilde{u}(\cdot, t)$ for all $t>t_{0}$ for any solution pair $u(\cdot, t), \tilde{u}(\cdot, t)$ with $u\left(\cdot, t_{0}\right) \geq \tilde{u}\left(\cdot, t_{0}\right)$ for some $t_{0} \geq 0[6]$. In 
particular, $\|u(\cdot, t)\|_{L^{1}\left(\mathbb{R}^{n}\right)}=|m|$ for all $t>t_{0}$ whenever $u_{0}$ does not change sign; otherwise, $\|u(\cdot, t)\|_{L^{1}\left(\mathbb{R}^{n}\right)}$ decreases monotonically in $t$ (Lemma 2.1), with $\|u(\cdot, t)\|_{L^{1}\left(\mathbb{R}^{n}\right)}>|m|$ for all $t$, therefore approaching a well defined asymptotic limit $\ell\left(u_{0}\right) \geq|m|$ for large $t$, whose value may be unknown [15]. A fundamental related question is whether equation (1.1) is asymptotic mixing, that is, the ergodic property

$$
\lim _{t \rightarrow \infty}\|u(\cdot, t)-\tilde{u}(\cdot, t)\|_{L^{1}\left(\mathbb{R}^{n}\right)}=0
$$

holds for any solutions $u(\cdot, t), \tilde{u}(\cdot, t)$ with the same mass: should (3.2) hold, it would immediately follow that $\ell\left(u_{0}\right)=|m|$ for any $u_{0} \in L^{1}\left(\mathbb{R}^{n}\right)$. One example is given by (1.6), for which property (3.2) is known to hold [22]. The problem

of identifying which equations (1.1) happen to be asymptotic mixing remains largely open.

\section{References}

[1] Aronson, D. G., Bounds for the fundamental solution of a parabolic equation, Bull. Amer. Math. Soc. 73 (1967), 890-896.

[2] Braz e Silva, P.; Zingano, P.R., Some asymptotic properties for solutions of one-dimensional advection-diffusion equations with Cauchy data in $L^{p}(\mathbb{R})$, C. R. Acad. Sci. Paris, Ser. I, 342 (2006), 465-467.

[3] Braz e Silva, P.; Schütz, L.; Zingano, P. R., On solution boundedness for parabolic equations, Far East J. Math. Sciences, 33 (2009), 379-385.

[4] Carlen, E. A.; Loss, M., Sharp constant in Nash's inequality, Internat. Math. Res. Notices, no. 7 (1993), 213-215.

[5] Carlen, E. A.; Loss, M., Optimal smoothing and decay estimates for viscously damped conservation laws, with application to the 2-D Navier-Stokes equation, Duke Math. J. 86 (1996), 135-157. 
[6] Crandall, M.; Tartar, L., Some relations between nonexpansive and order preserving mappings, Proc. Amer. Math. Soc., 78 (1980), 385-390.

[7] Escobedo, M.; Zuazua, E., Large time behavior for convection-diffusion equations in $\mathbb{R}^{n}$, J. Func. Anal. 100 (1991), 119-161.

[8] Escobedo, M.; Vázquez, J. L.; Zuazua, E., On a diffusion-convection equation in several space dimensions, Indiana Univ. Math. J. 42 (1993), $1413-$ 1440.

[9] Friedman, A., Partial Differential Equations, Holt, Rinehart and Winston, New York, 1969.

[10] Harabetian, E., Rarefactions and large time behavior for parabolic equations and monotone schemes, Comm. Math. Phys. 114 (1988), 527-536.

[11] Ilin, A. M.; Kalashnikov, A. S.; Oleinik, O. A., Second order linear equations of parabolic type, Russ. Math. Surv. 17 (1962), 1-143.

[12] Moser, J., A new proof of de Giorgi's theorem concerning the regularity problem for elliptic differential equations, Comm. Pure Appl. Math. 13 (1960), $457-468$.

[13] Moser, J., On Harnack's theorem for elliptic differential equations, Comm. Pure Appl. Math., 14 (1961), 577-591.

[14] Nash, J., Continuity of solutions of parabolic and elliptic equations, Amer. J. Math., 80 (1958), $931-954$.

[15] Rudnicki, R., Asymptotical stability in $L^{1}$ of parabolic equations, J. Diff. Eqs. 102 (1993), 391-401.

[16] Schonbek, M. E., Decay of solutions to parabolic conservation laws, Comm. Partial Diff. Eqs. 7 (1980), $449-473$.

[17] Schonbek, M. E., Uniform decay rates for parabolic conservation laws, Nonl. Anal. T. M. A. 10 (1986), 943-956. 
[18] Schütz, L., Some results about advection-diffusion equations, with applications to the Navier-Stokes equations (Portuguese), Doctorate Thesis, Universidade Federal do Rio Grande do Sul, Porto Alegre, Brazil, June 2008.

[19] Shatah, J., private communication, 1990.

[20] Zingano, P. R., Nonlinear stability analysis for two classes of waves, $\mathrm{PhD}$ dissertation, New York University, 1990.

[21] Zingano, P. R., Nonlinear $L^{2}$ stability under large disturbances, J. Comp. Appl. Math., 103 (1999), 207-219.

[22] Zingano, P. R., Asymptotic behavior of the $L^{1}$ norm of solutions to nonlinear parabolic equations, Comm. Pure Appl. Anal. 3 (2004), 151-159.

P. Braz e Silva

Departamento de Matemática Universidade Federal de Pernambuco Recife, PE 50740, Brazil

E-mail: pablo@dmat.ufpe.br
L. Schütz Instituto de Matemática, Estatística e Física Universidade Federal de Rio Grande Rio Grande, RS 96201, Brazil

E-mail: lineiaschutz@furg.br

P. R. Zingano

Departamento de Matemática Pura e Aplicada

Universidade Federal do Rio Grande do Sul

Porto Alegre, RS 91509, Brazil

E-mail: pzingano@mat.ufrgs.br 